\title{
Diagnostica per immagini nella nefrolitiasi
}

\author{
F.M. Drudi, R. Profiti, F. Marchetti
}

Dipartimento di Scienze Radiologiche, Università “La Sapienza”, Roma

\section{$\mathbf{L}$}

a nefrolitiasi è una patologia frequente, responsabile di un numero importante di ricoveri ospedalieri e di prescrizioni di esami radiologici, attuati sia per confermare la diagnosi che per stabilire un trattamento adeguato. La nefrolitiasi è una malattia multifattoriale, per cui è impossibile, nell'approccio a questa malattia prescindere dall'epidemiologia e dalla clinica (1). Il ruolo del diagnosta è quello di miscelare sapientemente due mondi apparentemente lontani tra loro: quello clinico e quello della tecnologia avanzata.

In generale le basi etiopatogenetiche e cliniche di una patologia sono gli strumenti che servono a colui che si cimenta nella diagnostica per sapere cosa cercare e dove cercare. Il miglior utilizzo di apparecchiature e tecniche, la profonda conoscenza delle macchine e le loro evoluzioni tecnologiche permettono di decidere come meglio cercare la patologia in esame. In altri termini posto il quesito clinico spetta al diagnosta scegliere il mezzo migliore e il più economico per raggiungere l'obiettivo prefisso.
L'epidemiologia della nefrolitiasi tende a variare con l'area geografica, con la condizione socio-economica, con la composizione dei calcoli e con la loro localizzazione nel tratto urinario, nella vescica o nei reni. A livello mondiale l'incidenza maggiore si risconta in Arabia Saudita 20\%, nel Nord America $13 \%$, nell'Europa 5$9 \%$ in Asia 1-5\% (2). Negli ultimi dieci anni la frequenza della nefrolitiasi in Italia è passata da 11.7 a 17.2 su 1,000 abitanti, 4 volte più frequente negli uomini che nelle donne, l'età di insorgenza e tra i 20 ed i 30 anni, con un un incremento nel sud Italia, e un decremento nel nord Italia. Un'incidenza maggiore si riscontra in soggetti con familiarità, con tendenza a mangiare cibi ricchi di proteine animali, e con un incremento dell'indice di massa corporea $(\mathrm{MBI})>32 \mathrm{~kg} / \mathrm{m}^{2}$ (3).

Il follow-up di pazienti che hanno formato un calcolo di sali di calcio, ha dimostrato che facilmente vanno incontro a recidive nello stesso lato o dal lato opposto. La recidiva ha la massima frequenza entro 2-3 anni. Il $50 \%$ dei pazienti presenta recidive entro 5 anni, il $60 \%$ entro 10 anni. Per tale motivo in tutti i pazienti che hanno emesso anche un solo calcolo di calcio, sono necessari controlli diagnostici periodici (1-2 anni).

Nei pazienti con sospetta colica renale l'iter diagnostico tradizionale è quello dell'esecuzione di un esame radiografico diretto dell' addome associato a un esame ecografico (4). L'esame diretto radiografico da solo è poco diagnostico in quanto non è in grado di visualizzare i calcoli di acido urico (radiotrasparenti), e con un diametro inferiore ai $4 \mathrm{~mm}$, ed inoltre non offre la certezza che l'immagine calcifica visualizzata sia di pertinenza delle vie urinarie se non quando il calcolo assume aspetti tipici (a "stampo" per i calcoli di struvite, a "bersaglio" per i calcoli vescicali o ovalari per i calcoli ureterali). L' ecografia visualizza i calcoli indipendentemente dalla loro composizione o origine, anche se di piccole dimensioni (4-5 mm con cono d' ombra posteriore, e 2-3 $\mathrm{mm}$ se è presente dilatazione delle vie escretrici a monte) e in tutte le sedi essi si trovino. Alcune difficoltà diagnostiche si possono presentare a livello della giunzione pielo-ureterale, 


\section{Giornale di Tecniche \\ Nefrologiche \\ \& Dialitiche \\ Anno XVI n ${ }^{\circ} 3$ \\ (C) Wichtig Editore 2004

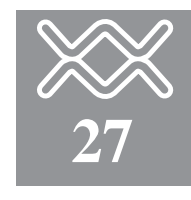

in quanto quest'ultima si presenta come un' area iperecogena, in cui un calcolo difficilmente viene visualizzato, tranne se non è causa di stasi e nel tratto lombare dell'uretere. L'associazione di queste due metodiche permette di riconoscere la patologia litiasica a sede reno-pelvica e a livello ureterale sottogiuntale e terminale; si rileva inoltre la stasi urinaria in caso di ostruzione. La dilatazione delle vie escretrici non pone abitualmente difficoltà diagnostiche, se si escludono alcune condizioni patologiche (distensione renale da iperdiuresi, replezione urinaria ecc).

Un ulteriore contributo all' ecografia è dato dal Color-Doppler con il "twinkling artefact" o artefatto da luccichio, che consente di confermare le immagini sospette. Questo artefatto consiste in un'area di colore rosso e blu che si proietta dorsalmente a strutture riflettenti come calcificazioni e calcoli. La possibilità di misurare con l'esame Doppler gli indici di resistenza intrarenali spiega meglio la sindrome ostruttiva, e le condizioni circolatorie del parenchima renale. La differenza degli indici di resistenza tra un rene ostruito e un rene normale è di 0.12 (0.71-0.59).

L'urografia è ormai impiegata solo prima del trattamento chirurgico endoscopico e di litotrissia, per lo studio morfologico e funzionale del rene, infatti rimane ancora l'indagine cardine, nella diagnosi di alcune patologie malformative delle vie escretrici renali che altre indagini radiologiche non sono in grado di documentare. La analisi morfologica ottenibile con l'esame urografico permette di valutare la dilatazione delle cavità escretrici, loro eventuali rotture e il contorno dei calcoli lungo la colonna opaca. In alcuni casi la dilatazione può essere assente in quanto i piccoli calcoli non modificano l'aspetto dell'uretere, ma i segni funzionali possono modificare la diagnosi (5).

L'evoluzione tecnologica ha messo a disposizione dei radiologi la TC spirale a singolo o multistrato, grazie a questa nuova apparecchiatura uno studio dell'addome e della pelvi si completa in circa 15 secondi e cioè con una sola e breve apnea inspiratoria. Nei pazienti con colica renale acuta la TC spirale, senza iniezione di mezzo di contrasto, è utilizzata come metodica di scelta svolgendo un ruolo fondamentale, come hanno dimostrato all'inizio del 1995 Smith et al. La TC del paziente con colica renale è basata sulla scoperta di segni diretti (presenza del calcolo, edema della giunzione uretero-vescicale, ostruzione ureterale ecc) e segni indiretti (idronefrosi ureterale, infiltrazione del grasso perirenale ecc), che permettono 1' identificazione non solo del calcolo, ma anche di altri segni che possono servire per la guida e la gestione del paziente e a valutare la prognosi a lunga scadenza. Tale metodica ha infatti la capacità sia di rilevare le anomalie extraurinarie che provocano dolore e simulano una colica renale sia di studiare l'intero decorso dell'uretere, identificando la posizione esatta della calcificazione con una valutazione simile a quella ottenuta con l'urografia tradizionale (6). Inoltre il parenchima renale dei pazienti che presentano ostruzione ureterale o segni indiretti di ostruzione ha un valore di attenuazione maggiore o uguale a $5 \mathrm{UH}$ rispetto al parenchima di un rene normale (7). Tutti i calcoli, anche quelli radiotrasparenti, sono iperdensi in TC, la sola eccezione è descritta nei soggetti HIV positivi trattati con l'indinavir un farmaco inibitore della proteasi (8). La principale difficoltà diagnostica consiste nella certezza di identificare i calcoli ureterali da altre immagini iperdense: calcificazioni vascolari e soprattutto fleboliti. Il passaggio spontaneo del calcolo attraverso le vie escretrici renali è in rapporto alle sue dimensioni infatti un calcolo di $1 \mathrm{~mm}$ di diametro ha circa 1'87\% di probabilità di essere espulso spontaneamente mentre un calcolo di 7-9 $\mathrm{mm}$ di diametro ha solo il $25 \%$ di probabilità. Ma dipende anche dalla sua localizzazione: $48 \%$ in sede prossimale, $60 \%$ in sede mediale e $75 \%$ in sede distale (9). Per cui è importante sapere ciò per stabilire un corretto follow-up del paziente. La scelta di un protocollo diagnostico non può prescindere dalla considerazione sulla disponibilità di apparecchiature, dai costi monetari e biologici. L'analisi dei costi mostra che la spesa della TC è uguale o poco inferiore al costo dell'urografia, ma la dose di radiazioni erogata al paziente è maggiore nell'esame TC. I protocolli impiegati infatti oscillano da valori di 2.82 e $6.4 \mathrm{mSV}$, incidendo in maniera preminente sulle gonadi dei soggetti di sesso femminile. Tale problematica ha spinto molti ricercatori a utilizzare protocolli cosiddetti "low-dose" (10), I protocolli "low dose" (mA 70, collimazione 5), hanno una sensibilità e specificità del 96 e $97 \%$ e un valore predittivo positivo e negativo del 99 e $90 \%$ rispettivamente. Questi risultati si avvicinano molto a quelli della TC a maggior esposizione di radiazioni, l'unico limite è rappresentato dai pazienti obesi per i quali la tecnica "low-dose" non è applicabile. Nella selezione dei pazienti a rischio maggiore di esposizione ai raggi $X$ (pazienti in gravidan$\mathrm{za}$, in età pediatrica ecc.) è in uso come indagine preliminare nello studio della colica renale acuta la Risonanza Magnetica. Questa tecnica permette una rappresentazione multiplanare dell' apparato urinario, è molto sensibile ai liquidi stazionari, e le sequenze urografiche permettono di visualizzare non solo l'uretere dilatato, ma anche l'infiltrazione edematosa perirenale. I calcoli appaiono come una struttura senza segnale all'estremità dell' uretere dilatato (11). L'utilizzo della $\mathrm{RM}$ riduce il numero di pazienti da sottoporre a studio TC e di conseguenza il carico di radiazioni, ma a oggi presenta costi maggiori, ha una minore diffusione sul territorio e non è scevra da falsi negativi. La sensibilità e la specificità delle diverse tecniche varia a seconda delle casistiche, ma si è concordi nell'attribuire una sensibilità che va dal $57 \%$ del radiogramma diretto dell'apparato urinario al $96 \%$ della TC con una specificità che varia dal $71 \%$ dell'esame radiografico diretto al $97 \%$ dell' ecografia e perfino al $100 \%$ dell' esame TC (12). In conclusione quale è la migliore panacea da proporre nella gestione del paziente affetto da colica renale? Molti autori sono d'accordo nel soste- 
nere quale metodica sostitutiva dell'esame radiologico l'ecografia, mentre l'urografia è da utilizzare solo nei casi complessi con possibili patologie associate. La cultura anglosassone, specie la scuola americana, vede nella Spiral CT il primo presidio diagnostico nel paziente con colica renale, è questa una tesi indubbiamente ben sostenibile ma non dimentichiamo due importanti cose, l'ecografia in quei paesi la effettuano dei tecnici (sonographers) e spesso bisogna giustificare l'acquisto di molte Spiral CT. Per cui credo che la migliore chiave di lettura per risolvere questo problema sia come sempre legata alla voglia di collaborare del clinico e del diagnosta, al contesto ospedaliero in cui si trovano ad operare e alla loro singola esperienza, l'uno per selezionare al meglio i veri pazienti con colica renale, l'altro per scegliere il miglior presidio diagnostico per risolvere il problema. Infine non dimentichiamo il ruolo centrale che svolge il protagonista di tutto e cioè il paziente, conoscere a fondo la storia clinica, i suoi dati laboratoristici, effettuare su di lui una corretta visita consente di meglio inquadrare la problematica e di conseguenza di trovare la migliore soluzione ai suoi problemi.

\section{BIBLIOGRAFIA}

1. Otal P, Irsutti M, Chabbert V, Murat C, Ducasse JL, Rousseau H, Joffre F. Radiologic study of renal colic. J. Radiol 2001; 82 (1): 27-33.

2. Ramello A, Vitale C, Marangella M. Epidemiology of nephrolithiasis. J Nephrol 2000; 13 (suppl) 3: S45-50.

3. Amato M, Lusini ML, Nelli F Epidemiology of nephrolithiasis today. Urol Int 2004; 72 (suppl) 1: 1-5.

4. Shokeir AA. Renal colic: news concepts related to patophisiology, diagnosis and treatment. Curr Opin Urol 2002; 12 (4): 263-9.

5. Haroun A. Duplex Doppler sonography in patient with acute renal colic: Prospective study and literature. Int Urol Nephrol 2003; 35 (2): 135-40.

6. Eshed I, Kornecki A, Rabin A, Elias S, Katz R. Unenhanced spiral CT for the assessment of renal colic. How does limiting the referral base affect the discovery of additional findings not related to urinary tract calculi? Eur J Radiol 2002; 41 (1): 60-4.

7. Goldman SM, Faintuch S, Ajzen SA, Christofalo DM, et al. Diagnostic value of attenuation measurements of the kidney on unenhanced helical CT of obstructive ureterolithiasis. AJR Am J Roentgenol 2004; 182 (5): 1251-4.

8. Blake SP, McNicholas MMJ, Raptopulos V. Non opaque crystal deposition causing ureteric obstruction in patients with HIV undergoing indinavir therapy. AJR 1998; 171: 717-20.

9. Colli DM, Varanelli MJ, Smith RC. Relationship of spontaneous passage of ureteral calculi to stone size and location as revealed by unenhanced helical CT. AJR 2002; 178: 101-3.

10. Meagher T, Sukumar VP, Collingwood J, et al. Low dose compu- ted tomography in suspected acute renal colic. Clin Radiol 2001; 56 (11): 873-6.

11. Otal PH, Irsutti M, Chabbert V, et al. Imaging of renal colic. J Radiol 2001; 82: 27-33.

12. Springhart WP, Preninger GM. Advanced imaging in stone management. Curr Opin Urol 2004; 14 (2): 95-8. 